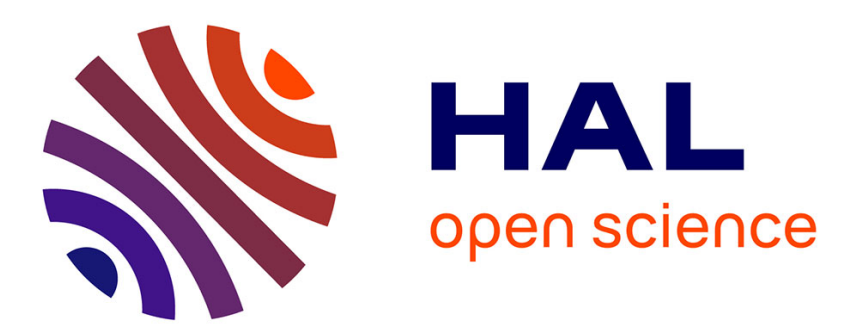

\title{
L'impact d'électrons lents sur les surfaces de matériaux diélectriques : méthodes d'analyse et applications industrielles
}

\author{
L. Sanche
}

\section{- To cite this version:}

L. Sanche. L'impact d'électrons lents sur les surfaces de matériaux diélectriques: méthodes d'analyse et applications industrielles. Journal de Physique IV Proceedings, 1997, 07 (C6), pp.C6-175-C6-187. 10.1051/jp4:1997615 . jpa-00255714

\author{
HAL Id: jpa-00255714 \\ https://hal.science/jpa-00255714
}

Submitted on 1 Jan 1997

HAL is a multi-disciplinary open access archive for the deposit and dissemination of scientific research documents, whether they are published or not. The documents may come from teaching and research institutions in France or abroad, or from public or private research centers.
L'archive ouverte pluridisciplinaire HAL, est destinée au dépôt et à la diffusion de documents scientifiques de niveau recherche, publiés ou non, émanant des établissements d'enseignement et de recherche français ou étrangers, des laboratoires publics ou privés. 


\title{
L'impact d'électrons lents sur les surfaces de matériaux diélectriques : méthodes d'analyse et applications industrielles
}

\section{Sanche}

Groupe CRM en Sciences des Radiations, Faculté de Médecine, Université de Sherbrooke, Sherbrooke, Québec, J1H 5N4, Canada

\begin{abstract}
Résumé : Les électrons de basse énergie ( $0-30 \mathrm{eV})$ sont impliqués en tant que source d'excitation primaire ou en tant que produits secondaires dans un grand nombre de processus industriels et de techniques d'analyse des matériaux. Leurs interactions près de la surface de solides diélectriques peut être étudiée en utilisant des faisceaux d'électrons de faible énergie à haute résolution. Les principales expériences permettant d'étudier ces interactions sont brièvement décrites dans cet article. Des exemples de résultats obtenus avec ces dernières sont inclus afin d'illustrer les mécanismes de base et de fournir une description des processus fondamentaux de dégradation impliqués durant lirradiation. L'importance de ces mécanismes pour la dosimétrie, le vieillissement électronique des câbles à haute tension et la nanolithographie est expliquée brièvement.
\end{abstract}

\begin{abstract}
Low energy electrons (0-30 eV) are involved in a large number of industrial processes and of analytical techniques for material analysis either as secondary particles or as the primary excitation source. The interaction of these electrons near the surface of dielectric solids can be investigated with highresolution low-energy electron-beam techniques. The major types of experiments are briefly described in this article and examples of the results obtained from them are given to illustrate the basic mechanisms which control the electron-solid interactions and to provide a description of the basic degradation processes involved during irradiation. The importance of these mechanisms for dosimetry, electronic aging of high-voltage cables and nanolithography is briefly explained.
\end{abstract}

\section{INTRODUCTION}

Depuis plus de deux décennies, plusieurs travaux théoriques et expérimentaux ont porté sur l'étude de l'interaction des électrons de basse énergie $(0-30 \mathrm{eV})$ avec la surface de matériaux diélectriques [1]. Grâce à ces travaux, nous connaissons maintenant mieux les mécanismes fondamentaux qui mènent à la création de phonons, à l'excitation vibrationnelle et électronique, à la dissociation moléculaire et au piégeage d'électrons lors d'un bombardement électronique. Ces mécanismes interviennent dans plusieurs réactions chimiques induites par la radiation ionisante ou directement par des électrons de faible énergie. Ces électrons interviennent donc dans certaines applications industrielles où ils sont produits directement ou par l'entremise d'un processus primaire.

Dans cet article, je mentionne d'abord en quoi l'étude de ces mécanismes est importante dans trois applications spécifiques: le vieillissement électronique des câbles à haute tension, la nanolithographie et la dosimétrie. Par la suite, les techniques d'analyse sont décrites et sont accompagnées de quelques résultats expérimentaux typiques reliés aux mécanismes d'interaction importants ainsi qu'aux applications mentionnées. 


\section{APPLICATIONS INDUSTRIELLES}

\subsection{Le vieillissement électronique des câbles à haute tension}

Le vieillissement électronique [2-4] dans l'isolant d'un câble à haute tension est le résultat de l'interaction d'un excès de porteurs non-thermalisés avec le matériau diélectrique, lequel induit des réactions chimiques thermiques ou de plus haute énergie. Les électrons chauds sont présentement considérés comme étant la source majeure de porteurs non-thermalisés qui contribuent de façon significative au claquage final $[4,5]$. Récemment, il a été admis que les excitations induites par les électrons chauds étaient responsables de la dégradation chimique dans les diélectriques organiques ayant été soumis à des champs électriques intenses [6].

Le rôle des électrons chauds (i.e. des électrons de faible énergie) dans le vieillissement électronique a récemment été révisé par l'auteur [7]. Lorsqu'un champ électrique intense est appliqué à un isolant, une partie des électrons piégés sont relâchés dans la bande de conduction, ceci résultant de l'absorption d'énergie provenant de la distribution thermique des phonons du solide ou de particules externes (par exemple, des photons U.V., des rayons $\gamma$ ) pénétrant dans le solide. Lorsque son énergie excédentaire peut être dissipée dans le diélectrique par l'intermédiaire des pertes d'énergie causées par l'excitation des phonons et par des excitations vibrationnelles intra-moléculaires, l'électron libéré est généralement incapable de gagner suffisamment d'énergie cinétique pour demeurer dans la bande de conduction en tant que particule libre ou quasi-libre. La répétition de ce processus donne naissance à un courant dit "de fuite". Toutefois, d'un point de vue statistique, il existe toujours une probabilité pour que certains électrons, à un temps donné, gagnent suffisamment d'énergie cinétique pour exciter les niveaux d'énergie électroniques des constituants moléculaires du diélectrique. Si certains de ces états électroniques sont dissociatifs, les molécules se décomposent en libérant des radicaux libres extrêmement réactifs, lesquels sont fortement susceptibles d'induire d'autres réactions et de changer la nature chimique du solide. Certaines de ces réactions peuvent contribuer à la formation de défauts, lesquels peuvent être vus comme des pièges inter et intramoléculaires (ou atomiques) pour les électrons et pour les trous.

Cette image simplifiée du vieillissement électronique est utile pour en expliquer les principes de base, bien qu'elle n'illustre pas certaines situations pratiques de manière adéquate. En réalité, les électrons de basse énergie peuvent induire des modifications chimiques plus facilement aux interfaces solide-solide, solide-gaz et solide-vide. En effet, dans le solide, les effets de cage peuvent souvent prévenir la dissociation moléculaire et les recombinaisons de charges et de fragments sont hautement probables tandis qu'aux interfaces, le contact de différents matériaux favorise les réactions chimiques et empêche la recombinaison des charges. De plus, un électron chaud traversant une interface solide-gaz (par exemple, un électron entrant dans une cavité située à l'intérieur d'un isolant électrique) peut être accéléré dans l'espace gazeux où, grâce à la faible densité de la matière présente, il lui est plus probable d'atteindre l'énergie cinétique requise pour causer la dissociation moléculaire et la formation de piègès. On s'attend à ce que les électrons chauds accélérés dans des cavités par des champs électriques intenses possèdent des énergies situées entre 0 et $15 \mathrm{eV}$ [8] lorsqu'ils pénètrent à nouveau le solide. Par conséquent, leurs effets à l'intérieur de diélectriques soumis aux forces d'un champ intense peuvent être bien représentés lors d'expériences décrivant l'action d'électrons monochromatiques $(0-15 \mathrm{eV})$ provenant du vide et bombardant une surface diélectrique. Ce genre d'expériences est brièvement décrit dans la section III de cet article. L'action des électrons de basses énergies joue donc un rôle important dans le vieillissement des câbles de transmission d'énergie soumis à des tensions élevées et ce, particulièrement au niveau du transport en courant continu pour lequel la plupart des contraintes électromécaniques sont éliminées. 


\subsection{La nanolithographie}

Les récentes découvertes sur les possibilités d'accroître, de provoquer et même de contrôler des réactions chimiques à l'aide de photons et de faisceaux de particules bombardant des surfaces ont favorisé un intérêt scientifique croissant pour l'étude de la dynamique des réactions non-thermiques déclenchées par ces particules. On s'attend à ce que les méthodes photoniques et par faisceaux d'électrons puissent être utilisées en nanolithographie et même qu'elles surpassent les techniques macroscopiques actuelles (par exemple, la gravure au plasma) dans l'industrie des dispositifs de micro-électronique exigeant une grande précision [9].

Les techniques photoniques impliquent l'utilisation de photons laser ayant des énergies situées entre $0,1 \mathrm{eV}$ dans l'infrarouge à environ $6 \mathrm{eV}$ dans l'ultraviolet [9-11]. On a montré que plusieurs des réactions optiquement induites sur une surface sont causées par la photogénération d'électrons de basse énergie, lesquels transfèrent leur charge et de l'énergie aux molécules adsorbées, causant ainsi la dissociation et/ou la désorption [11-15]. On s'attend également à ce que les électrons de faible énergie jouent un rôle dominant dans les mécanismes physiques et chimiques fondamentaux impliqués dans les techniques de microlithographie utilisant de la radiation de haute énergie telles que l'écriture directe (avec un faisceau d'électrons) et la gravure (avec des rayons $X$ générés par laser ou par synchrotron [16]). En fait, les électrons de haute énergie et les photons transfèrent la plupart de leur énergie au matériau exposé en produisant une grande quantité $\left(\sim 4 \mathrm{X} 10^{4} / \mathrm{MeV}\right)$ d'électrons secondaires de basse énergie $(0-70 \mathrm{eV})$ ayant une énergie moyenne d'environ $10 \mathrm{eV}$ [17]. Les électrons secondaires diffusent dans le matériau irradié et à sa surface, là où ils peuvent interagir avec les molécules adsorbées pour produire des espèces hautement réactives telles des radicaux libres, des cations et des anions. Ces intermédiaires peuvent subséquemment réagir dans le voisinage de la surface du matériau irradié et changer sa nature chimique à l'intérieur de l'échelle de résolution spatiale de l'instrument.

Une application directe de limpact électronique à basse énergie à la nanolithographie apparaît dans l'utilisation du microscope à effet tunnel (MET), utilisé pour initier des réactions chimiques de surface [1821]. Dans ce cas, la pointe du MET, placée dans le voisinage de la surface, est utilisée comme source d'électrons pour produire des modifications chimiques locales et ce, avec une précision de l'ordre du nanomètre [18-21]. Il est possible d'induire la chimisorption par la rupture des liens intramoléculaires des molécules adsorbées [22]. De plus, une sélectivité chimique est possible par induction de réactions par attachement dissociatif résonant de l'électron incident. À titre d'exemple, des résultats sur la fluorination du Si hydrogéné amorphe recouvert de $\mathrm{CF}_{4}$ physisorbé sont présentés dans cet article. Ils démontrent qu'il est possible d'induire cette modification chimique de la surface par un faisceau d'électrons ayant des énergies inférieures au premier niveau électronique de $\mathrm{CF}_{4}$, ce qui limite considérablement le dommage, les espèces réactives et les réactions possibles avec la surface $\mathrm{Si}-\mathrm{H}$.

\subsection{La dosimétrie en radiobiologie}

Les ruptures double-brin dans l'ADN intranucléaire sont généralement considérées comme étant les précurseurs des effets biologiquement néfastes des radiations ionisantes dans les cellules de mammiferes. Si un instrument ayant une réponse de détection simulant celle de l'ADN des cellules peut être conçu, alors le dispositif sera apte à mesurer l'efficacité biologique absolue des radiations [23]. En d'autres termes, la quantité mesurée sera directement proportionnelle à l'effet des radiations chez l'humain et non pas seulement, comme c'est présentement le cas, à l'énergie absorbée par le tissu biologique. Un système unifié de dosimétrie ne requérant pas la connaissance à priori du type de rayonnement deviendrait possible et les conclusions ne dépendraient d'aucun modèle spécifique de l'action des radiations.

En pratique, une seconde génération d'instruments ayant une réponse basée sur un nouveau principe conceptuel s'avère nécessaire. Ces détecteurs pourraient être basés sur le principe voulant que les 
lésions "fatales" de l'ADN proviennent de bris multiples locaux (i.e. à l'intérieur d'un rayon d'environ $4 \mathrm{~nm}$ ) sur cette molécule [24]. Il nous faudrait donc construire des détecteurs capables de mesurer les dépôts multiples d'énergie à l'intérieur de telles dimensions. Présentement, la théorie et les recherches expérimentales effectuées avec un disque semi-infini de phosphore dopé au fluor, d'une épaisseur de 20 $\mu \mathrm{m}$, révèlent une méthode potentiellement prometteuse. Toutefọis, des études plus détaillées sont nécessaires sur l'optimisation de la concentration de fluor et sur le processus d'extraction du signal désiré à partir du dispositif pratique [25]. L'étalonnage du signal nécessitera une connaissance précise des libres parcours moyens de réaction des électrons secondaires à l'intérieur du dispositif avec les constituants de l'ADN afin de relier cette mesure physique à l'effet biologique.

\section{MÉTHODES D'ANALYSE}

L'interaction des électrons de basse énergie près de la surface d'un solide diélectrique peut être étudiée par impact d'électrons monoénergétiques sur un film multicouches. Ce film peut être fabriqué dans un système à hypervide par la condensation de gaz ou de vapeurs sur un substrat de métal propre maintenu à des températures cryogéniques ( 15 à $100 \mathrm{~K}$ ). Selon le type d'expérience, il est possible de mesurer, en fonction de l'énergie des électrons incidents, le courant transmis à travers le film condensé, les charges qui y sont piégées [26] ou le courant qui y est réfléchi [1]. Il est également possible de mesurer la dépendance énergétique des flux d'ions positifs, d'ions négatifs et d'espèces neutres émanant de la surface du film [7]. En général, l'épaisseur du film doit être plus grande que le libre parcours moyen total de l'électron si nous désirons minimiser les effets du substrat métallique. De petites quantités de molécules peuvent être ajoutées à la surface du film ou mélangées à l'intérieur du film diélectrique pour étudier l'effet d'impuretés. Les interactions des électrons à l'interface de deux matériaux diélectriques peuvent être étudiées en couvrant un film donné avec une couche d'une autre substance. Ces différents types d'expériences sont brièvement décrites dans cette section.

Un schéma du genre d'appareil utilisé pour enregistrer des spectres de transmission d'électrons de basse énergie et des rendements de désorption électroniquement stimulée est montré à la figure 1 . Il consiste en un canon d'électrons à haut courant (D), un spectromètre de masse quadripolaire (E), un volume calibré pour l'introduction des gaz (C), une cible froide (B) et un monochromateur trochoïdal à haute résolution (A). Selon linstrument, il peut être possible de faire tourner la cible (B) et de la refroidir, à l'aide du cryostat $(\mathrm{F})$, jusqu'à $15 \mathrm{~K}$. Toutes les composantes montrées à la Figure 1 sont logées dans un système à hypervide atteignant des pressions d'environ $10^{-8} \mathrm{~Pa}$. Le faisceau d'électrons, collimaté par un champ magnétique, quitte le monochromateur avec une résolution d'environ $40 \mathrm{meV}$ (largeur à mi-hauteur), et frappe le film condensé sur un substrat de métal (B) (la cathode). Ce dernier est électriquement 


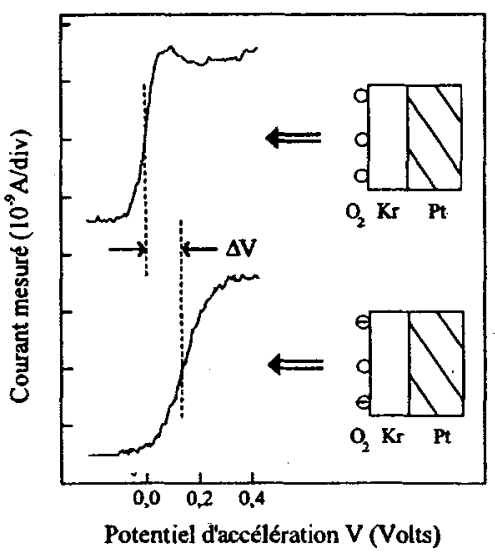

Figure 2 Courant transmis à travers un film de Kr non-chargé (courbe supérieure) et chargé (courbe inférieure), couvert de 0,1 monocouche de $\mathrm{O}_{2}$, en fonction du potentiel d'accélération $\mathrm{V}$ du faisceau incident d'électrons. Les charges sont créées par la réaction $\mathrm{e}^{-}+\mathrm{O}_{2} \rightarrow \mathrm{O}_{2}^{-} \rightarrow \mathrm{O}^{-}+\mathrm{O}$.

isolé du cryostat par un disque de saphir et est connecté à des conducteurs électriques (G). Des spectres de transmission d'électrons de basse énergie sont obtenus en mesurant le courant $I_{t}(E)$ arrivant au substrat de métal en fonction de l'énergie incidente des électrons. Dans ces expériences, $I_{t}$ est de l'ordre du nano-ampère et l'échelle d'énergie absolue des électrons est étalonnée à $0,15 \mathrm{eV}$ par rapport au niveau du vide en mesurant l'injection de la transmission électronique à travers les films. Le substrat est généralement une feuille de métal polycristallin pouvant être nettoyée par chauffage résistif au moyen des conducteurs électriques $(G)$. Les films condensés sont fabriqués en utilisant une procédure de dosage par expansion d'un volume gazeux pouvant être calibrée en enregistrant les effets quantiques d'épaisseur observés pour des films ultra-minces [1]. Avec cet étalonnage, des épaisseurs de film allant de 1 à $50 \mathrm{~nm}$ peuvent généralement être estimées avec une précision de $30 \%$, en supposant que la croissance du film se fasse couche par couche.

La spectroscopie de transmission d'électrons de faible énergie permet également de mesurer le nombre de charges accumulées près de la surface d'un film diélectrique après un bombardement électronique [26]. Lorsque les électrons provenant du monochromateur (A) (Figurel) ont juste assez d'énergie pour entrer dans un film multicouches déposé sur le substrat (B), une montée abrupte, nommée la courbe d'injection (CI), est observée dans le spectre de transmission. La CI pour un film non chargé est représentée par la courbe supérieure de la figure 2. Lorsque le même film est chargé à la surface par le faisceau d'électrons, la $C I$ est décalée de $\Delta V$ vers un potentiel d'accélération plus élevé (courbe inférieure, Figure 2) puisque les électrons incidents doivent posséder plus d'énergie cinétique pour franchir la barrière de potentiel négative. La CI est également élargie, ceci étant dû aux effets des distributions spatiales de charges et de densité de courant. De telles mesures sont généralement effectuées conjointement avec tous les types d'expériences faites avec des électrons de basse énergie sur des films minces afin de s'assurer que la cible ne se charge pas de façon significative durant les expériences. Toutefois, si le film est délibérément chargé à sa surface par une différence de potentiel $\Delta \mathrm{V}$ significative, celle-ci peut être reliée à la section efficace de piégeage si le film diélectrique est traité comme un condensateur [26]. La barrière de potentiel $\Delta V$ est reliée à la densité de charges $\sigma(t)$ accumulées après un temps de bombardement $t$, par les relations

$$
\begin{gathered}
\Delta V(t)=\sigma(t) \frac{h}{\varepsilon}, \\
\sigma(t)=\sigma_{0}(1-\exp [\beta t]), \beta=\frac{\rho J_{0}}{\mathrm{e}},
\end{gathered}
$$

où $\varepsilon$ est la permittivité du film, h est son épaisseur, $\sigma_{0}$ la densité initiale $(t=0)$ de pièges, $\rho$ la section efficace de piégeage, $J_{0}$ la densité de courant incident sur le film et e l'unité de charge. Dans la limite où $t \rightarrow 0$, un coefficient de charge $A=d \Delta V / d t$, directement proportionnel à la section efficace de piégeage, peut s'exprimer comme:

$$
\left.\frac{d \Delta V}{d t}\right|_{t=0}=\left[\frac{h \sigma_{0} J_{b}}{\varepsilon e}\right] \rho=A_{s} \text {. }
$$


Voici la façon dont l'expérience est effectuée. La CI d'un film multicouches fraîchement déposé est d'abord enregistrée très rapidement (par exemple, durant $0,1 \mathrm{~s}$ ) de façon à éviter de charger la cible de manière significative. Le film est alors bombardé à une tension donnée $\mathrm{V}$ appliquée entre le monochromateur et le film pour une période beaucoup plus longue (par exemple, $25 \mathrm{~s}$ ) avec le même courant incident (i.e. $\mathrm{I}_{0}=5 \times 10^{-9} \mathrm{~A}$ ). Par la suite, la $\mathrm{CI}$ est de nouveau rapidement enregistrée et le décalage $\Delta \mathrm{V}$ est déterminé par comparaison avec la CI initiale. Un tel cycle peut être répété plusieurs fois sur le même film avec le même $V$ pour obtenir la dépendance temporelle du processus. Un nouveau film doit être déposé pour chaque point mesuré lorsque nous désirons évaluer la dépendance en fonction de l'épaisseur du film et de l'énergie des électrons. Lorsque la charge produit un décalage $\Delta \mathrm{V}$ inférieur à la résolution du faisceau, des mesures en fonction de l'énergie des électrons incidents peuvent être faites sur un seul film sans affecter considérablement la résolution totale en énergie de l'expérience.

Une partie des ions positifs et négatifs produits par impact électronique sur la cible B (figure 1) peut être mesurée en plaçant un spectromètre de masse (E) près de la surface du film [27]. Dans le montage expérimental de la figure 1 , le cryostat $C$ est tourné vers $E$-pour effectuer cette mesure. Les ions sortant du film sont focalisés par des lentilles situées devant l'entrée du spectromètre de masse. Des grilles peuvent être insérées entre les lentilles et le spectromètre de masse de façon à analyser les énergies des ions par la méthode des potentiels retardateurs. L'appareil peut être utilisé selon deux modes [27]: le mode de rendements en ions dans lequel les ions d'une masse donnée sont détectés en fonction de l'énergie incidente des électrons, et le mode d'énergie des ions dans lequel le courant d'ions d'une masse donnée est mesuré pour une énergie fixe des électrons en fonction du potentiel retardateur. Dans des systèmes plus sophistiqués, il est aussi possible de mesurer la distribution angulaire de radiation U.V. émise ainsi que des espèces métastables et des ions désorbés par impact électronique. Dans ce genre d'appareil [28], les particules chargées peuvent être filtrées ou analysées en énergie par un ensemble de grilles concentriques autour de la cible. Ces particules, passant à travers la grille, sont subséquemment accélérées sur un dispositif d'amplification et d'imagerie. Les espèces neutres peuvent ètre mesurées en repoussant, à l'aide des grilles, toutes les particules chargées.

Les énergies perdues par les électrons près de la surface de films minces et leur dépendance énergétique sont mesurées par un spectromètre de perte d'énergie d'électrons à haute résolution [1]. Le montage expérimental est similaire à celui montré sur la figure 1 , les composantes $\mathrm{A}$ et $\mathrm{E}$ étant remplacées par deux déflecteurs électrostatiques hémisphériques. L'un d'eux produit un faisceau focalisé d'électrons monochromatiques, frappant la surface à un angle $\theta_{0}$ par rapport à la normale du film. L'autre analyse en énergie les électrons qui sont diffusés hors de la cible à un angle $\theta_{r}$ par rapport à la normale du film. Selon le type de spectromètre, les angles $\theta_{0}, \theta_{\mathrm{r}}$ ou les deux peuvent être variès à l'intérieur des limites imposées par l'arrangement physique de l'appareil (par exemple, dans notre appareil, $\theta_{0}=14^{\circ}$ à $70^{\circ}$ ). Des spectres de perte d'énergie d'électrons sont enregistrés en balayant l'énergie du monochromateur ou de l'analyseur. La dépendance de l'intensité d'une perte en fonction de l'énergie incidente de l'électron (c'est-à-dire la fonction d'excitation) est obtenue en balayant l'énergie des deux déflecteurs avec une constante différence de potentiel entre eux correspondant à la perte d'énergie sondée.

\section{DIFFUSIONS QUASI-ÉLASTIQUES ET EXCITATIONS VIBRATIONNELLES}

Le ralentissement des électrons de la bande de conduction dans les diélectriques est principalement dû à la création de phonons et/ou d'excitations vibrationnelles. Les études les plus directes de pertes d'énergie d'électrons pour l'excitation de phonons dans des diélectriques ont été effectuées par des expériences de transmission d'électrons de basse énergie, par spectroscopie par perte d'énergie d'électrons à haute résolution [7] et par photo-injection [6]. Il se trouve que de telles pertes d'énergie dépendent de la densité d'états de la bande de conduction et de l'interaction électron-phonon. La densité d'état de la bande de conduction calculée pour l'argon solide (ligne pleine) et celle extraite de spectres de transmission 


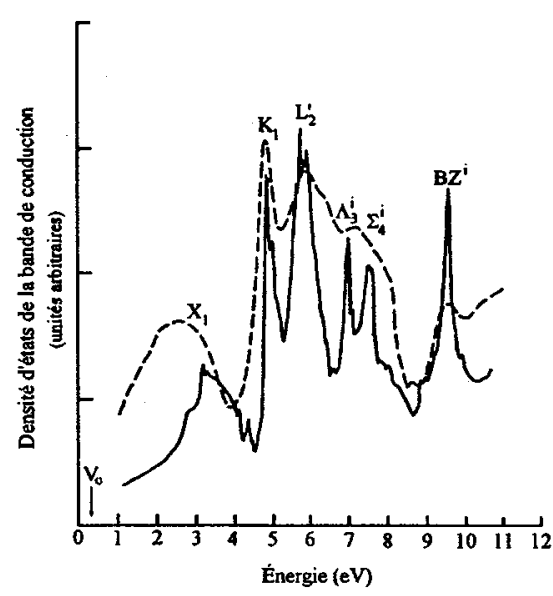

Figure 3 Densité d'états de la bande de conduction de l'Argon solide: calculée (trait continu) et déterminée à partir de l'analyse de données de spectres de transmission d'électrons de faible énergie dans l'argon à $\sim 20 \mathrm{~K}$. Le zéro d'énergie est celui du niveau du vide. $\mathrm{V}_{\mathrm{o}}$ est l'énergie du bas de la bande de conduction. d'électrons de faible énergie de films multi-couches (entre 20 et 100 monocouches) (tirets) [29] sont comparées sur la figure 3. Il existe une relation évidente entre les deux ensembles de données indiquant que c'est essentiellement la densité d'états de la bande de conduction qui gouverne la diffusion quasi-élastique des électrons de basse énergie (c'està-dire les pertes d'énergies pour l'excitation de phonons). Des expériences récentes de spectroscopie par perte d'énergie d'électrons à haute résolution [30] ont confirmé cette relation.

Des spectres de transmission d'électrons de basse énergie [31] d'un film de 5 monocouches de n-hexane enregistré à différentes températures (entre 15 et $70 \mathrm{~K}$ ) sont montrés à la figure 4 . Le large pic centré autour de $11 \mathrm{eV}$ est causé par des électrons ayant perdu de l'énergie pour produire des excitations électroniques. En-dessous de $8 \mathrm{eV}$, seules les diffusions quasi-élastiques sont possibles. Ainsi, les caractéristiques du spectre de transmission reflètent les structures de la densité d'états de la bande de conduction. Tel que montré sur la Figure 4, ces caractéristiques sont dépendantes de la température et indiquent qu'au-dessus de $60 \mathrm{~K}$, un état cristallin du solide commence à se former; à $70 \mathrm{~K}$, l'état cristallin semble être bien établi. Au-dessous de $40 \mathrm{~K}$, un état plus désordonné ou amorphe est prédominant. Puisque le plus bas niveau de conduction $\mathrm{V}_{0}$ du n-hexane se situe à $0,8 \mathrm{eV}$ au-dessus du niveau du vide, le pic saillant près de $0 \mathrm{eV}$ est causé par des électrons se situant dans la bande interdite, tel que montré par Caron et al. [32].

Lorsque la chaîne de l'hydrocarbure est plus longue, les spectres de transmission demeurent essentiellement les mêmes, tel que montré sur le spectre de la figure 5, enregistré par Ueno et al. [33]. Les courbes sur cette figure représentent les spectres de transmission à la température de la pièce pour des films cristallins de $n-\mathrm{C}_{11} \mathrm{H}_{64}, \mathrm{n}-\mathrm{C}_{36} \mathrm{H}_{74}, \mathrm{n}-\mathrm{C}_{44} \mathrm{H}_{90}$ et de polyéthylène dont les épaisseurs se situent approximativement entre 50 et $100 \AA$. Les pics identifiés de $\mathrm{A}$ à $\mathrm{F}$ sur le spectre de transmission du $\mathrm{n}-\mathrm{C}_{44} \mathrm{H}_{90}$ ont les mêmes caractéristiques que ceux observés sur le spectre de n-hexane à $70 \mathrm{~K}$. Ils ne sont toutefois pas aussi bien définis, ceci étant dû à la résolution de la source d'électrons dans l'expérience de Ueno et al. [33]. Leur présence peut être observée facilement en enregistrant la première dérivée selon l'énergie du spectre de transmission (courbe pointillée à la figure 5). La courbe pleine en haut de la figure 5 représente le spectre de transmission d'un film de polyéthylène de faible densité à la température de la pièce. Bien que les principales caractéristiques du spectre de transmission des alcanes

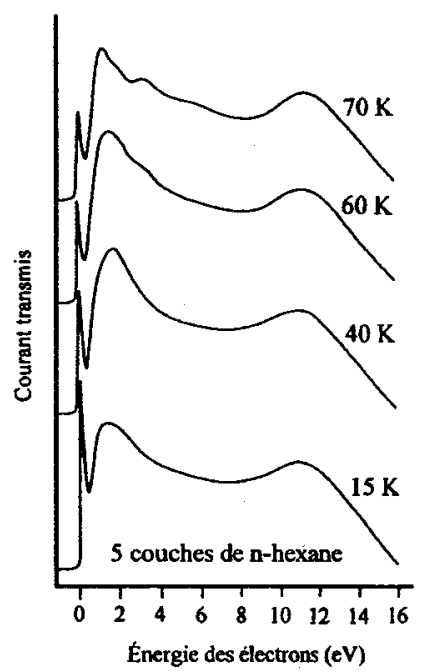

Figure 4 Spectres de transmission d'électrons de faible énergie à travers un film de 5 monocouches de n-hexane enregistré à différentes températures. Le courant au maximum de chaque courbe est de l'ordre de $5 \times$ $10^{-10}$ A. Le courant maximum arrivant au substrat de métal est aussi de cet ordre de grandeur. 


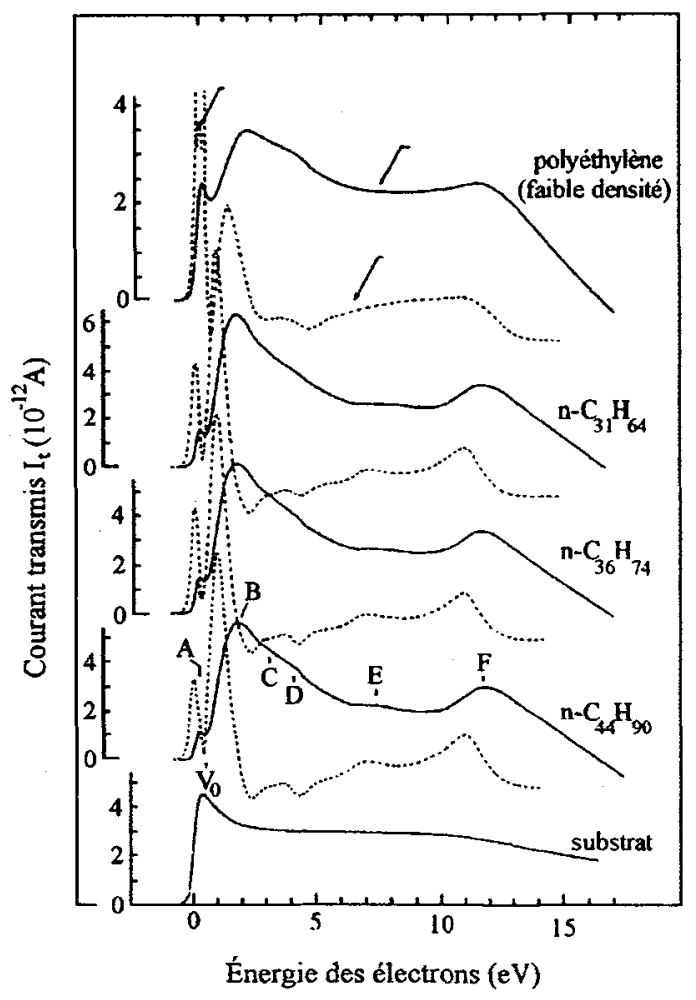

Figure 5 Spectres de transmission d'électrons de faible énergie à travers des films de $n-\mathrm{C}_{31} \mathrm{H}_{64}, n-\mathrm{C}_{36} \mathrm{H}_{74}, \mathrm{n}-\mathrm{C}_{44} \mathrm{H}_{90}$ et de polyéthylène à faible densité reproduits de la référence [33]. Les courbes pointillées représentent les dérivées énergétiques premières des spectres. Le courant mesuré sur le substrat est montré au bas de la figure. Les bas de la bande de conduction $\left(V_{0}\right)$ est indiqué par la flèche verticale. à longue chaîne soient préservées, le spectre du polyéthylène à faible densité ressemble davantage à celui enregistré à $60 \mathrm{~K}$ sur un film de $\mathrm{n}$-hexane. Ce résultat suggère que le film de polyéthylène de faible densité à la température de la pièce peut exister dans un état combiné, ayant des composantes cristallines et des composantes désordonnées, ou amorphes. De façon plus générale, les résultats des figures 4 et 5 indiquent que les caractéristiques principales de la densité d'état de la bande de conduction sont pratiquement indépendantes de la longueur de la chaîne pour les alcanes linéaires ayant plus de 5 atomes de carbone. On peut alors suggérer que de tels alcanes soient utilisés comme modèles du polyéthylène dans des études impliquant les effets de la densité d'état de la bande de conduction, tels la diffusion quasi-élastique. Cette possibilité est intéressante pour l'étude des mécanismes fondamentaux du vieillissement électronique de câbles à haute tension munis d'une gaine isolante de polyéthylène.

La figure 6 illustre un exemple d'excitation de modes vibrationnels de l'état fondamental de constituants moléculaires d'un diélectrique. L'expérience a été effectuée sur un film de $5 \mathrm{~nm}$ de $\mathrm{N}_{2}$ [34] avec $\theta_{0}=14^{\circ}$ et $\theta_{\mathrm{r}}=4^{\circ}$. Les spectres de perte d'énergie d'électrons ont été enregistrés pour des électrons ayant des énergies incidentes de 2,9,10,8 et 19,8 eV. Les gains verticaux de chaque courbe ou de chaque portion de courbe sont calculés par rapport au pic élastique. Chaque pic d'énergie perdue sur la figure 6 peut être associé à une excitation vibrationnelle de $\mathrm{N}_{2}$ dans l'état fondamental. On peut voir qu'à 2,9 et à $19,8 \mathrm{eV}$, les intensités des pertes d'énergie vibrationnelles sont grandement amplifiées (c'est-à-dire jusqu'à 2 ordres de grandeur pour les harmoniques). Cette augmentation est attribuable aux résonances de forme ${ }^{2} \Pi_{\mathrm{g}}$ et ${ }^{2} \Sigma_{\mathrm{u}}$ [34] (c'est-à-dire à la formation d'anions transitoires $\mathrm{N}_{2}-$ composés de $\mathrm{N}_{2}$ dans son état fondamental avec un électron occupant respectivement une orbitale $\pi$ et $\sigma$, orbitale habituellement vide de la molécule). On s'aperçoit que la formation d'un anion transitoire peut être extrêmement efficace dans le ralentissement des électrons chauds, par transfert de l'énergie de ces derniers à des excitations vibrationnelles. 


\section{DISSOCIATION MOLÉCULAIRE ET PIÉGEAGE DE L'ÉLECTRON}

Au-dessus d'un certain seuil énergétique, les électrons incidents sur des molécules isolées ou condensées peuvent causer une rupture des liens internucléaires, ce qui engendre une fragmentation. Tout dépendant de l'état intermédiaire impliqué et des processus d'échange de charge, ces fragments peuvent être neutres, positivement ou négativement chargés. Lors d'expériences sur les films moléculaires, les produits de la dissociation peuvent être mesurés par des techniques de désorption électroniquement stimulée telles que décrites dans la section 2. Les particules chargées peuvent ne pas posséder suffisamment d'énergie cinétique pour contrer la force de polarisation qu'elles induisent dans le solide. Celles qui sont incapables de quitter le solide peuvent être détectées en mesurant la section efficace de piégeage, tel que décrit à la section 2. Des exemples d'expériences avec des hydrocarbures et des fluorocarbures sont donnés dans cette section. Comme la formation d'anions transitoires est le processus dominant aux basses énergies dans les solides moléculaires [7], ces exemples sont limités à l'ạtachement électronique dissociatif (c'est-à-dire la dissociation d'un ion négatif transitoire en une particule neutre et un fragment négativement chargé).

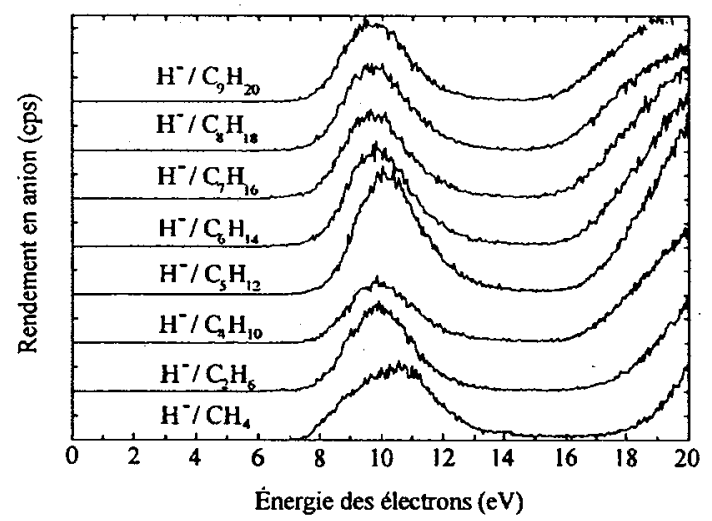

Figure 7: Distribution des rendements pour la formation de $\mathrm{H}^{-}$ dans des films épais (de 6 à 8 monocouches) d'hydrocarbones saturés déposés sur un substrat de platine et bombardés avec des électrons ayant une énergie située entre 0 et $20 \mathrm{eV}$. Pour une meilleure vue d'ensemble, les courbes sont décalées verticalement. Chaque division représente $2000 \mathrm{cps}$.

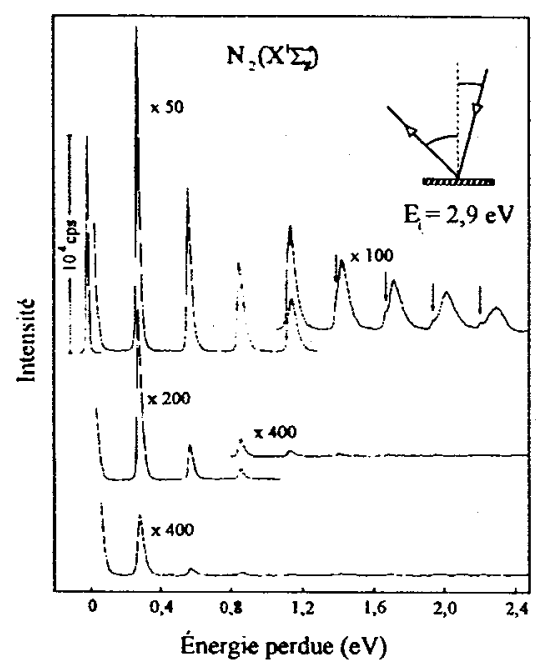

Figure 6 Spectres de perte d'énergie d'électrons d'un film multicouches désordonné de $\mathrm{N}_{2}$ enregistrés à des énergies de faisceau de 2,9, 19,8 et 10,8 eV. L'intensité du pic élastique est indiquée en comptes par secondes (cps). L'augmentation des excitations vibrationnelles de l'état fondamental à 2,9 et à $19,8 \mathrm{eV}$ résulte de la formation d'ions négatifs transitoires $\mathrm{N}_{2}{ }^{-}$dans les configurations ${ }^{2} \Pi_{\mathrm{g}}$ et ${ }^{2} \Sigma_{\mathrm{g}}{ }^{+}$, respectivement

Les rendements d'anions spécifiques qui désorbent de divers films d'hydrocarbures en fonction de l'énergie incidente des électrons et de l'épaisseur du film ont été fournis par Rowntree et al. [35]. Le plus grand rendement en anion observé était invariablement celui de $\mathrm{H}^{-}$, et ce pour tous les hydrocarbures saturés et pour toutes les épaisseurs de film. Chaque distribution de rendement en $\mathrm{H}^{-}$d'alcanes saturés a montré un seul pic résonant près de $10 \mathrm{eV}$ d'énergie incidente des électrons, ceci étant causé par la dissociation de $\left(\mathrm{C}_{n} \mathrm{H}_{2 \mathrm{n}+2}\right)^{-}$en fragments de $\mathrm{C}_{n} \mathrm{H}_{2 \mathrm{n}+1}+\mathrm{H}^{-}$ , le seuil de formation de $\mathrm{H}^{-}$étant situé près de $7 \mathrm{eV}$. La figure 7 montre, pour des épaisseurs de film de 6-8 monocouches, un ensemble typique de fonctions de rendement en $\mathrm{H}^{-}$pour chacun des n-alcanes étudiés. $\grave{A}$ 


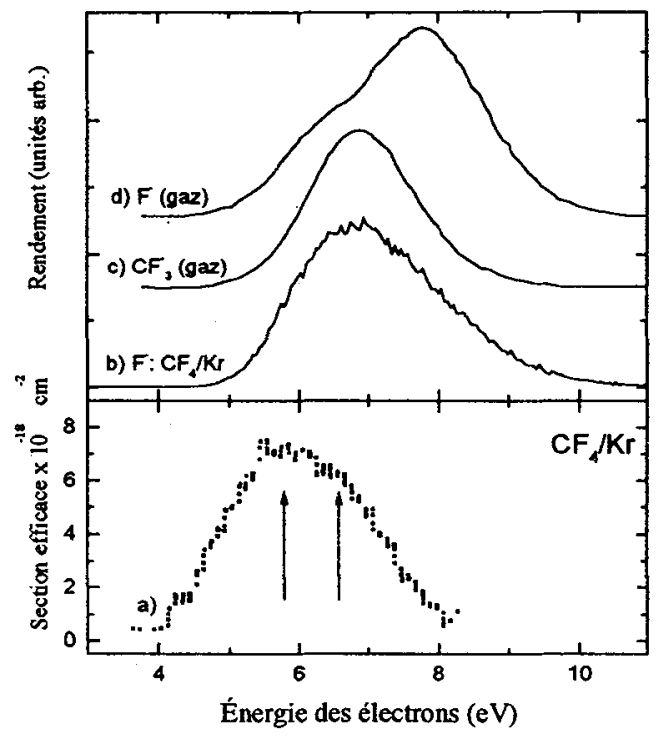

Figure 8: a) Section efficace de piégeage pour un film de 0,2 monocouche $(\mathrm{MC})$ de $\mathrm{CF}_{4}$ condensé sur un film de $15 \mathrm{MC}$ de $\mathrm{Kr}$. b) Rendement en $\mathrm{F}^{-}$pour $0,15 \mathrm{MC}$ de $\mathrm{CF}_{4}$ sur $15 \mathrm{MC}$ de $\mathrm{Kr}$. c) et d) Rendements de $\mathrm{CF}_{3}{ }^{-}$et $\mathrm{F}^{-}$, respectivement, dans la phase gazeuse.

l'exception du méthane, tous les rendements en $\mathrm{H}^{-}$provenant de l'attachement dissociatif ont une largeur à mi-hauteur d'environ 2,3$2,5 \mathrm{eV}$, lesquels sont relativement constants pour des épaisseurs de film supérieures à 3 monocouches. De plus, pour les alcanes ayant plus de 5 atomes de carbone, l'intensité, l'énergie et la forme du signal sont pratiquement inchangées avec la longueur de la chaîne. Il s'ensuit donc que le n-hexane et les alcanes à plus longue chaine peuvent possiblement être utilisés comme modèles du polyéthylène en ce qui concerne les études de production de l'anion d'hydrogène et de la dissociation moléculaire au-dessous de $14 \mathrm{eV}$. Au-dessus de cette énergie, chacun des alcanes produit un flux de $\mathrm{H}^{-}$faible mais non-nul, qui augmente graduellement en intensité au-dessus d'environ $15-18 \mathrm{eV}$, tel que montré à la figure 7 . Ce flux est dû à la dissociation dipolaire :

$$
\left.\left[\left(\mathrm{C}_{\mathrm{n}} \mathrm{H}_{2 \mathrm{n}+2}\right)^{*} \rightarrow\left(\mathrm{C}_{\mathrm{n}} \mathrm{H}_{2 \mathrm{n}+1}\right)^{+}+\mathrm{H}^{-}\right)\right]
$$

Un autre exemple de dissociation moléculaire par attachement dissociatif est montré sur la courbe b) de la figure 8 pour une petite quantité $\left(0,15\right.$ monocouche) de $\mathrm{CF}_{4}$ déposé sur un film de 15 monocouches de $\mathrm{Kr}$ [36]. Cette courbe représente la dépendance énergétique du rendement de $\mathrm{F}^{-}$désorbé de la surface du film. La figure 8a) montre comment la section efficace de piégeage, mesurée par la méthode décrite à la section 2 , varie en fonction de l'énergie incidente des électrons. Les rendements d'ions $\mathrm{CF}_{3}-$ et $\mathrm{F}^{-}$en phase gazeuse sont montrés sur les figures 8c) et 8d), respectivement. Dans la phase gazeuse, l'intensité relative des deux fragments [37] est $\mathrm{F}^{-}: \mathrm{CF}_{3}{ }^{-}=1: 0,6$. On voit sur la figure 8 qu'il existe des similarités entre ces quatre courbes et que les phénomènes de désorption électroniquement stimulée et de piégeage électronique, observés dans la phase condensée, décrivent essentiellement le même processus d'attachement dissociatif observé dans la phase gazeuse. Toutefois, en regardant de plus près les données de la phase gazeuse, on observe quelques différences. Alors que la formation de $\mathrm{CF}_{3}{ }^{-}$est associée à un profil de type Gaussien autour de $6,8 \mathrm{eV}$, le spectre de $\mathrm{F}^{-}$présente un maximum à $7,6 \mathrm{eV}$ et a une structure près de $6,8 \mathrm{eV}$. Ce comportement a été interprété comme étant la capture de l'électron dans deux états d'ion négatif: l'état fondamental $\mathrm{CF}_{4}{ }^{-}$et l'état électroniquement excité $\mathrm{CF}_{4}{ }^{*}-[38,39]$. L'état fondamental de l'anion se décompose selon les surfaces d'énergie potentielle correspondant aux canaux complémentaires

$$
\begin{gathered}
\mathrm{CF}_{4}^{-}(6,8 \mathrm{eV}) \rightarrow \mathrm{F}+\mathrm{CF}_{3}^{-} \\
\mathrm{CF}_{4}^{-}(6,8 \mathrm{eV}) \rightarrow \mathrm{F}^{-}+\mathrm{CF}_{3},
\end{gathered}
$$

tandis que les états excités fournissent exclusivement $\mathrm{F}^{-}$. En conséquence, le rendement en $\mathrm{F}^{-}$est composé de contributions provenant de ces deux résonances superposées. En plus de produire du $\mathrm{F}^{-}$, il est probable que le $\mathrm{CF}_{4}{ }^{*-}$ se dissocie pour former un radical excité $\mathrm{CF}_{3}{ }^{*}$ qui lui-même se dissocie subséquemment [38]:

$$
\mathrm{CF}_{4}^{*-}(7,6 \mathrm{eV}) \rightarrow \mathrm{F}^{-}+\mathrm{CF}_{2}
$$




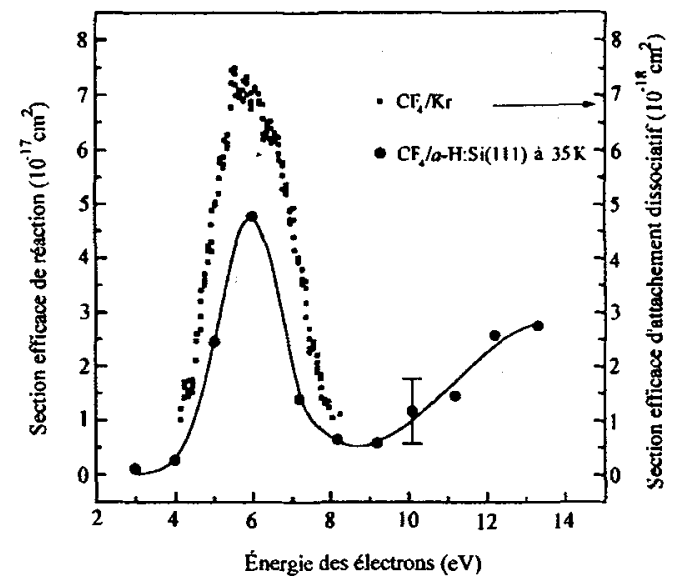

Figure 9: Section efficace expérimentale pour la fluorination de la surface $a-S i(111)$ avec le $\mathrm{CF}_{4}$ physisorbé en fonction de l'énergie incidente des électrons. Pour une meilleure vision des résultats, une courbe pleine relie les données. Le résultat de la section efficace totale d'attachement dissociatif $\Leftrightarrow$ pour le $\mathrm{CF}_{4}$ condensé sur du $\mathrm{Kr}$ est tracé à partir de la Figure 8.

(1) efficace maximale de piégeage est $7,3 \times 10^{-18} \mathrm{~cm}^{2}$. Elle est plus grande que la section efficace d'attachement dissociatif en la phase gazeuse par un facteur situé entre quatre et sept. En fait, les valeurs de la Figure 8a) sont des limites inférieures puisqu'une fraction des ions $\mathrm{F}^{-}$désorbe du film [36]. Comme aucun signal significatif de charge n'a été observé au-dessous de $4 \mathrm{eV}$ et entre 8 et $11 \mathrm{eV}$ au cours de cette expérience, nous pouvons conclure que le mécanisme principal de piégeage au-dessous de $11 \mathrm{eV}$ pour ce système est l'attachement dissociatif. Ce processus ne dissocie pas seulement de façon efficace la molécule de $\mathrm{CF}_{4}$, avec une section efficace d'environ $10^{-17} \mathrm{~cm}^{2}$, mais il crée également un ion $F^{-}$sur la surface de $\mathrm{Kr}$ (i.e. un piège profond).

L'attachement dissociatif à la molécule de $\mathrm{CF}_{4}$ a pu être utilisé pour déposer du fluor à la surface d'un semi-conducteur. Les résultats [40] de la figure 9 montrent la section efficace de fluoration partielle d'une surface de $\mathrm{Si}(111)$ amorphe $(a-\mathrm{H}: \mathrm{Si}(111))$ à $35 \mathrm{~K}$ induite par lirradiation d'une couche de tétrafluorure de carbone physisorbée avec des électrons de 2-13 eV. Les sections efficaces de réaction dépendent fortement de l'énergie des électrons incidents et ont un seuil à $4,0 \mathrm{eV}$ et une résonance évidente centrée à $6,0 \mathrm{eV}$, d'une valeur maximale de $4,8 \times 10^{-17} \mathrm{~cm}^{2}$. Cette dernière est attribuée à l'attachement dissociatif à la molécule de $\mathrm{CF}_{4}$ et démontre la sélectivité énergétique de l'attachement dissociatif pour modifier chimiquement une surface de semi-conducteur. Ici, la chimisorption du fluor sur la surface du $\mathrm{Si}$, produisant le lien Si-F, a été mesurée par spectroscopie de photoélectrons $\mathbf{X}$. Contrairement aux réactions utilisant des électrons de hautes énergies, le substrat et l'adsorbat ne sont pas endommagés sauf par I'unique réaction désirée, c'est-à-dire $\mathrm{e}^{-}+\mathrm{CF}_{4} \rightarrow \mathrm{CF}_{4}^{-} \rightarrow \mathrm{F}^{-}+\mathrm{CF}_{3}$ et $\mathrm{F}+\mathrm{CF}_{3}^{-}$et $\mathrm{F}^{-}+\mathrm{F}+\mathrm{CF}_{3}$ qui mène à la chimisorption du fluor (c'est-à-dire à la formation d'un lien Si-F). Cette observation suggère qu'en choisissant les adsorbants ayant les résonances appropriées pour l'attachement dissociatif, nous pourrions efficacement induire des réactions spécifiques sur les surfaces de semi-conducteurs par irradiation avec des électrons de faible énergie. À l'aide d'un faisceau d'électrons hautement localisé tel celui émanant de la pointe d'un microscope à effet tunnel, il devrait être possible d'induire une fluoration partielle d'une surface de Si sur l'échelle du nanomètre par attachement dissociatif. 


\section{Remerciements}

Ce travail a été financé par le Conseil de Recherches Médicales du Canada, Hydro-Québec et le CRSNG. L'auteur désire remercier Marie-Josée Fraser pour l'aide apportée dans la préparation de cet article.

\section{Références}

[1] Pour une revue, voir Sanche L. (Excess Electrons in Dielectric Media, CRC Press, C. Ferradini et J.-P Jay-Gerin, éditeurs, 1991) Chapitre 1.

[2] Zeller H. R., Baumann Th., Cartier E., Derschy H., Pfluger P. et Stucki F., "The physics of electrical breakdown and prebreakdown in solid dielectrics", Advances in Solid State Physics (Festkörperprobleme) (1987) 27, pp. 223-240.

[3] Cartier E. et Pfluger P., "Detection of hot-electron induced radiation damage in orgasnic dielectrics by exoelectron emission from thin films", IEEE Trans. on Electrical Insulation, El-22 (1987) pp.123-128.

[4] Pfluger P., Cartier E. et Dersch H., "Electron transport in thin insulating films; physics and effects on dielectric aging", Proc. of the IEEE Int. Symp. on Electrical Insulation, Cambridge, MA 91988) p. 135.

[5] Zeller H. R., Pfluger P. et Bernasconi J., "High-mobility states and dielectric breakdown in polymeric dielectrics", IEEE Trans. on Electrical Insulation, El-19 (1984) pp. 200-204.

[6] Marsolais R. M., Cartier E. A. et Pfluger P., (Excess Electrons in Dielectric Media, CRC Press, C. Ferradini et J.-P Jay-Gerin, éditeurs, 1991) Chapitre 2.

[7] Sanche L., IEEE Trans. on Electrical Insulation, 28 (1993) 789.

[8] Pour des cavités vides, le gain énergétique est proportionnel au volume. Dans le cas de cavités gazeuses, le gain devient limité par la diffusion des électrons dans le gaz au fur et à mesure que la taille de la cavité augmente. D'après les facteurs énergétiques de Townsend, on estime que cette limite peut être aussi élevée que $15 \mathrm{eV}$ pour des gaz à la pression atmosphérique. Voir McDaniel E. W., Collision Phenomena in Ionized Gases, (J. Wiley \& Sons, New York, 1964) Chapitre 11.

[9] Corcoran E., Sci. Am., 263 (1990) 98.

[10] Chuang T. J., Matter. Res. Soc. Proc., 17 (1983) 45; 29 (1984) 185; Surf. Sci. 178 (1986) 178 et références citées.

[11] Pour une revue des travaux publiés sur la chimie induite par les photo-porteurs par excitation d'un substrat semi-conducteur, voir Lu Z., Schmidt M. T., Podlesnik D. V,, Yu C. F. et Osgood R. M. Jr., J. Chem. Phys., 93 (1990) 7951.

[12] Marsh E. P., Gilton T. L., Meirer W., Schneider M. R. et Cowin J. P., Phys. Rev. Lett. 61 (1988) 2725; Marsh E. P., Tabares F. L., Schneider M. R., Gilton T. L., Meirer W. et Cowin J. P., J. Chem. Phys., 92 (1990) 2004.

[13] Zhu X.-Y., Hatch S., Campion A. et White J. M., J. Chem. Phys. 91 (1989) 5011; Zhou X.-L. et White J. M., Chem. Phys. Lett., 167 (1990) 205; Zhou X.-L., Zhu X.-Y. et White J. M., Acc. Chem. Res. 23 (1990) 327 et références citées.

[14] Hasselbrink E., Jakubith S., Nettesheim S., Wolf M., Cassuto A. et Ertl G., 3154; Hatch S. R., Zhu X.-Y., White J. M. et Campion A., J. Chem. Phys., 92 (1990) 2681.

[15] Buntin S. A., Richter L. J., Cavanagh R. R. et King D. S., Phys. Rev. Lett., 61 (1988) 1321.

[16] Anderson S. G., Komeda T., Seo J. M., Capusso C., Waddell G. D., Benning P. J. et Weaver J. H., Phys. Rev. B 42 (1990) 5082.

[17] Pour une revue du rôle des électrons de basse énergie dans la physique et la chimie des radiations, voir Sanche L., Radiat. Phys. Chem., 32 (1988) 269.

[18] Dagata J. A., Scheir J., Harary H. H., Evans C. J., Postck M. T. et Bennett J., Appl. Phys. Lett., 56 (1990) 2001. 
[19] Dujardin G., Walkup R.E. et Avouris Ph., Science, 255 (1992) 1232.

[20] Avouris Ph. et Lyo I. W., Surf. Sci., 242 (1992) 1.

[21] Wiesendanger R., J. Vac. Sci. Technol., B12 (1994) 515.

[22] Ehrich G., Activated Chemisorption in Chemistry and Physics of Solid Surfaces VII (Springer Verlag, 1988) p.1.

[23] Watt D. E., J. Radiol. Prot. 9 (1989) 33.

[24] Ward J. F., Progress in Nucleic Acid Research and Molecular Biology, 35 (1988) 95.

[25] Watt D. E., Radiat. Prot. Dosim. 27 (1989) 73.

[26] Marsolais R. M., Deschênes M. et Sanche L., Rev. Sci. Instr., 60 (1989) 2724.

[27] Azria R., Parenteau L. et Sanche L.; J. Chem. Phys., 8 (1987) 2292.

[28] Leclerc G., Bass A. D., Mann A. et Sanche L., Phys. Rev. B, 60 (1992) 4865.

[29] Plenkiewicz P., Jay-Gerin J.-P., Plenkiewicz B. et Perluzzo G., Sol. State Comm., 57 (1986) 203.

[30] Michaud M., Cloutier P. et Sanche L., Phys. Rev. B, 44 (1991) 10485.

[31] Sanche L. et Perluzzo G., non publié.

[32] Caron L. G., Perluzzo G., Bader G. et Sanche L., Phys. Rev. B, 33 (1986) 3027.

[33] Ueno N., Sugita K., Seki K. et Inokuchi H., Phys. Rev. B, 34 (1986) 6386.

[34] Sanche L. et Michaud M., Chem. Phys. Lett., 84 (1981) 497.

[35] Rowntree P., Parenteau L. et Sanche L., J. Phys. Chem., 95 (1991) 4902.

[36] Bass A. D., Gamache J., Parenteau L. et Sanche L., J. Phys. Chem., 99 (1995) 6123.

[37] Harland P. W. et Franklin J. L., J. Chem. Phys., 61 (1974) 1621,.

[38] Illenberger E., Chem. Phys. Lett., 80 (1981) 153.

[39] Oster T., Kuhn A., Illenberger E., Int. Mass. Spectrom. Ion Processes, 89 (1989) 1.

[40] Di W., Rowntree P. et Sanche L., Phys. Rev B, 52 (1995) 15. 